\author{
Military Technical College \\ Kobry El-Kobbah, \\ Cairo, Egypt.
}

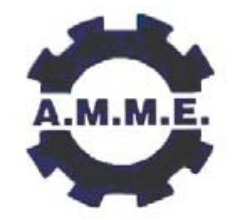

\title{
MODELING AND IMPLEMENTATION OF THE EGYPTIAN RAILWAYS TRACKING AND CONTROL SYSTEM
}

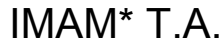

\begin{abstract}
Egypt Government decided to implement a new advanced Railways Tracking and Control System (RTCS). The main aim is to protect the citizen's (passengers) life, future, and business through achieving quick trains-conflict detection, and resolution, short response times in the event of disasters and other operational disturbances, and elimination of most or all "human factors" trains accidents. TRCS can also overcome the problem of railways traffic congestion which has increasingly become a major economic barrier to the flow of goods across the country. A team has been dedicated for the Egyptian RTCS project where the system investigation, data gathering, feasibility study, data analysis, system design phases have been accomplished and the project is now in the real implementation phase. The new advanced RTCS combines real-time rescheduling with an advanced train control tool. The system is designed to increase railway network capacity and stability in heavily used mixed traffic networks while minimizing the cost of building new railway infrastructure. The system combines real-time rescheduling, train control, and a new strategy for train operations into a coordinated Main Control Center (MCC) for traffic management. This approach is based on saturated use of network capacity bottlenecks, which is implemented by ensuring that MCC and every train always have up-to-date, conflict free schedule containing time, and speed and route information. The implementation of the Egyptian RTCS shall provide the input data about the railway subsystem (i.e. stations, locomotives, trains, trips...etc) that help in modeling the RTCS. The objectives of the modeling are to assign the fleet of locomotives to a network of trains while satisfying a variety of trips constraints and minimizing the total economic cost.
\end{abstract}

The research on the modeling of railways transportation include scheduling theory, graph theory, mathematical programming, operational theory, and some have addressed the problem of traffic controlling from multiple levels' points of view. In this paper, RTCS planning is formulated as Mixed Integer Programming (MIP) problem. The modeling network is a weekly space-time network where arcs denote trains, nodes denote events (arrivals and departures of trains at terminals), and different locomotive types define different commodities.

The objectives of this paper are to present the building of Egyptian RTCS project effort and to emphasis on the benefits of implementing this project as well as to present the use of integer MIP techniques for modeling such an RTCS.

\section{KEY WORDS}

Railways Tracking and Control System, Operational research, transportation problem, linear programming, decision making, system Modeling.

* Al Azhar University, Faculty of Science for Girls 


\section{INTRODUCTION}

Railways Tracking and Control System (RTCS) is an integrated command, control, communications, and information system for controlling train movements with safety, security, precision, and efficiency. RTCS is comprised of digital data link communications networks, continuous and accurate positioning systems such as Differential Global Positioning System (DGPS), On-Board Computers (OBC) with digitized maps on locomotives and maintenance-of-way equipment, in-cab displays, throttle-brake interfaces on locomotives, wayside interface units at switches and wayside detectors, and control center computers and displays. RTCS is interfaced with train traffic planners, work order reporting systems, and locomotive health reporting systems. RTCS issues movement authorities to train and maintenance-of-way crews, track the location of the trains and maintenance-of-way vehicles, have the ability to automatically enforce movement authorities, and continually update operating data systems with information on the location of trains, locomotives, cars, and crews. The remote intervention capability of RTCS will permit the control center to stop a train should the locomotive crew be incapacitated. In addition to providing a greater level of safety and security, RTCS also enables a railroad to run scheduled operations and provide improved running time, greater running time reliability, higher asset utilization, and greater track capacity. It will assist railroads in measuring and managing costs and in improving energy efficiency.

RTCS is a concept, rather than a single technology. RTCS is concerned with the systems and methods for controlling the movement of locomotives (and their trains) through a multiple route railway system with improved efficiency and safety. Locomotives differ both in horsepower, which affects their speed, and their "adhesion" (or tractive effort) which affects their ability to get a train moving from source to terminal stations. High-adhesion, high-powered locomotives can move the most freight. The importance of horsepower versus adhesion depends on the grade for a particular train. When assigning locomotives to trains, the first issue that has to be considered is how much power is needed to pull the train. A train might require 2.2 horsepower per ton. Locomotives movements are precisely monitored and orchestrated in accordance with a dynamic schedule that is determined through an evaluation of delivery requirements, coordination among all locomotives, speed restrictions and the effects of the track topography and locomotives grouping on locomotives response to brake and power application.

It can include many different capabilities, covering a range of railroad functions.

Examples of potential benefits from building RTCS include:

- line capacity enhancement

- improved service reliability

- faster over-the-road running times

- more efficient use of cars and locomotives (made possible by real-time location information)

- reduction in locomotive failures (due to availability of real-time diagnostics) of larger "windows" for track maintenance (made possible by real-time location information)

- fuel savings.

Railways grouping of the infrastructure (railway network routes), trips, trains, and 
locomotives. The locomotives are of various types; each type has different characteristics such as horsepower, tractive effort (or pulling) capacity, and costs. The locomotive modeling problem is to assign a consist (a set of locomotives) to each train in a preplanned train schedule so as to provide each train with sufficient power to pull the locomotives from its origin to its destination. Generally, a consist is chosen from a set of different fleet types. For example, a train's consist may be composed of two locomotives of types one and one locomotive of type two. The consist should be assigned to each train in a pre-planned weekly train schedule. This schedule should ensure that each train in the weekly train schedule gets sufficient power to pull its load and that the total cost of locomotive usage is minimized. The resulting plan must honor a variety of business rules, cannot require more locomotives than what is available in the total fleet, and must result in a plan that is relatively simple and repeatable. In this paper, we present the use available operation research solvers such as QSB+ (Qualification System for Business Plus) and MSIS (Management Science Information System) which include linear and non linear programming, transportation problem, and decision making to model the railway tracking and control. The rest of this paper is organized as: following the introduction in Section 1, Section 2 presents implementation of the Egyptian RTCS project; Section 3 provides an overview of the RTCS modeling functional model. The model was then defined in Section 4. In Section 5, mentioning the input that shall be collected from the Egyptian RTCS and output data expected from the RTCS. Finally, the paper was concluded in Section 6.

\section{IMPLEMENTATION OF THE EGYPTIAN RTCS PROJECT}

The objectives of the project goods and services concerning the implementation, installation, configuration, setup, training, technical support, and maintenance of the Egyptian railway for locomotive management functionality for 450 mobile units.

The three components of the Egyptian RTCS (Fig.1.) are:

1) The On-Board Computer (OBC) (or device or unit) which is considered as the " train black box" with built-in DGPS location capability,

2) The communication gateway that has the task to bring data from the air to the Railways Main Control Center (MCC), in our case we used the cellular wireless digital data link between the locomotives and the MCC.

3) The central office hardware and software at the control center which composed of the Database server, the Geographical Information System (GIS) with detailed maps of the Egyptian Railways routes, and the Graphical User Interface (GUI).

The real time localization of locomotives and trains allow to precisely knowing anytime where locomotives are. Quickly and effectively communicate with staff, making it possible to get quick and precise decisions. The wide range of mobile communications (Global System for Mobile Communications (GSM), General Packet Radio Service (GPRS), and Enhanced Data for GSM Evolution (EDGE)) supported by the three incountry mobile operators allows to choose the best and more efficient communication channel for every application. The accurate sampling of railway roads. Locomotives and trains allow the railway decision makers to evaluate all the costs and to control problems concerning some locomotives (anomalies).

The large amount of internal memory and the Operating System efficiency in data 


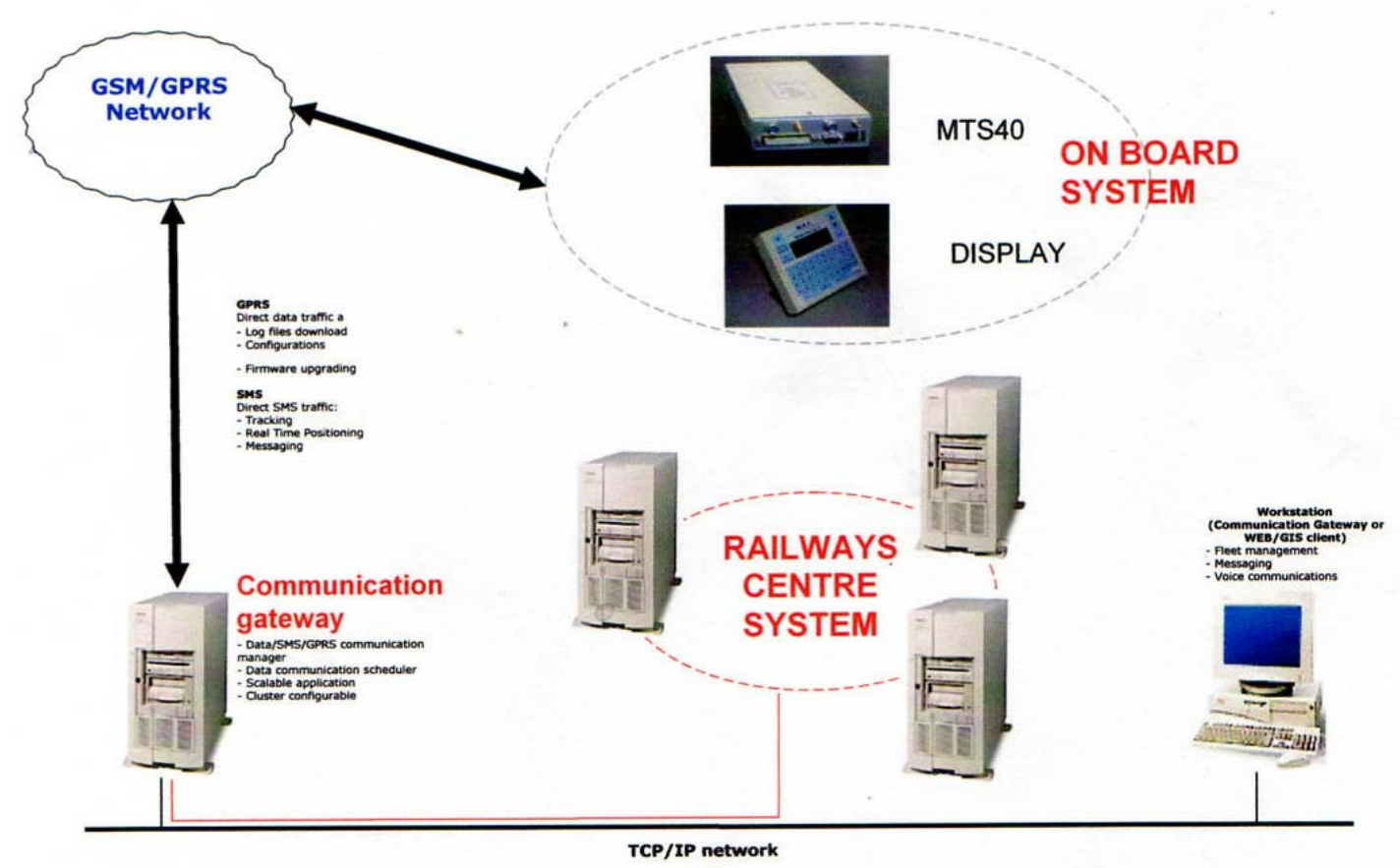

Fig.1. Components of the Egyptian RTCS, MTS40 is the OCB

storage, allows to record all the data needed: for example the locomotive positions (up to 12.000 points) can be logged every 4 seconds and any event (up to 1.000) selected as relevant (over-speed, alarm detection, trash can weighing, driver recognition, cargo temperature, etc.) is logged.

Sensors and on-board equipments can be easily interfaced, allowing the use of the on board hardware even for specific applications such for Cooling Chain Management (temperature sensors), Waste Management (weighing systems), Police Forces, Fire Brigade and Emergency Health Services. Quick evaluation of routes to be done by locomotives, with a remarkable reduction on the expenses needed to organize fleet and sensible reduction of tours distances and costs due to fleet organization. All the data related to driving/standing time and kilo-meter-age is also automatically recorded.

Through use of the cellular digital data link and real-time train location information, RTCS can be a train control system. The digital data link and the OBC are used for positive safety enforcement, stopping trains if movement authorities are exceeded. The same data link is also used for voice communications to transmit work instructions to train crews, receive acknowledgment of completed work, or transmit locomotive diagnostic information in real time.

The RTCS shall be implemented in two phases: RTCS-A then RTCS-B. RTCS-A is the "overlay" system that provides enforcement of movement authorities, but does not incorporate a "vital" central safety system. Existing train control methods (signals and/or voice radio) remain in use, with RTCS-A providing positive enforcement (e.g., trains are stopped before they exceed authority limits). RTCS-A rely solely on DGPS for location. Since RTCS-A incorporates the digital radio link and OBC, it can also be used to issue "work orders" (instructions to train crews regarding the pick-up and delivery of freight 
cars), transmit locomotive diagnostics and record crew on-duty and off-duty times and train delays. Provision of real-time train location and speed to train dispatchers via the digital data link has been presumed to improve dispatching effectiveness, reducing train delays and increasing line capacity.

RTCS-B shall include the vital central safety system. This function requires more precise location information than RTCS-A since it includes the use of accelerometers and a gyroscope (depending on the locomotive types) that give locomotives the ability to resolve location down to a particular track. In this case, the distance between following trains is reduced to that required to stop the following train short of a rear-end collision.

The real-time location information provided by the RTCS enables railroad managers to exercise more effective control of locomotives and freight cars, increasing asset productivity and provide the capability to issue instructions ("work orders") to train crews in real time. These instructions direct crews to deliver or pick up freight cars; RTCS also permits the crews to report the completion of this work in real time.

Again, this permits more effective management of rail equipment. The digital data link is used to report diagnostic data on locomotives in real time, allowing diagnose malfunctions and order necessary parts before a locomotive arrives in the workshop. Diagnostics also should provide warning of impending failures, possibly allowing train crews to take actions that avoid an en-route failure that delays trains. Real-time data on train location and speed also will allow track maintenance forces (track inspectors and others) to more effectively utilize their time.

Finally, real-time position information will allow train dispatchers to "pace" trains between scheduled meet points, permitting fuel savings. Current practice is to run trains at authorized speeds, often arriving at meet points well ahead of schedule. With realtime information on the location of opposing trains, it may be possible to slow a train down to save fuel while still arriving on schedule at the meet point.

\section{DGPS the Central Functionality of RTCS}

GPS is used to provide accurate, real-time position information to railroad operating personnel on a continuous basis. This required that the locomotive have a GPS receiver on board. That receiver would listen for signals continuously from a constellation of GPS satellites in geosynchronous orbit over the Earth.

By receiving signals simultaneously from at least four such satellites, it is possible to determine position within 100 meters. While that information can be combined with data on railroad track location to develop a more detailed and precision location, that level of position accuracy was not always sufficient for safety purposes. More accurate positioning is now possible using Differential GPS (DPGS). By establishing known locations throughout the country and placing GPS receivers and transmitters at those locations, a "correction" signal can be broadcast over a local area, making it possible to use GPS for determining which track in multiple-track territory a train is operating on. DGPS provide access to accurate territory maps with resolution to 3 meters, continually updated. 


\section{RTCS Core Functions and Hardware / Software}

RTCS Core Functions and Hardware / Software include all subsystems necessary to implement the following functions:

- Have latest-generation Geographic Information System (GIS) and Computer Aided Design (CAD) system to generate authorities (a more distributed system is an option)

- Positive train stops enforced for trains.

- Flexible block capability, but only where capacity constraints exist (will require additional communications bandwidth).

- Adequate warning time for highway grade crossing still in place for high-speed operations (>100 km/h.)

- Track circuits or an alternative technology for broken rail protection

- Alert "nearby" trains when emergency braking applied.

\section{Train (On board segment)}

\section{Mobile communications}

Mobile communications are provided by the local mobile operators that provide data and voice communications with Short Message System (SMS) and GPRS as part of their second and third generation (G2 and G3) technologies. High availability through Service Level Agreements (SLA) shall guarantee for the Egyptian RTCS, where authentication, authorization, and encryption of messages will be necessary.

\section{Man-Machine Interface (MMI)}

Powerful workstations are used with keyboard or Touch Screen; optionally, display capable of providing location information, warning annunciation, and means to input train crew responses. OBC system to work with office or field based systems to control authority issuance and release process. Same computer could also be used as part of positioning solution. MMI must have capability to provide all information to train crew necessary for RTCS operations including:

- Authority Limits

- Track Integrity / Switch Position

- Intended Route Information

- Braking Profile Margins for preventing unnecessary interventions. (Knowledge of impending positive enforcement)

- Location Information

- Displaying relative position of other location-broadcasting track vehicles (for use in ensuring positive separation between track forces and trains)

- MMI must provide straightforward capability for train crew inputs

- Information displayed on MMI must be validated to be correct (vital closed loop operation)

- MMI visible for all crew members, through separate MMls for each railway authorization zone.

\section{On-Board Computer OCB System}

$\mathrm{OBC}$ is required to process a large amount of information necessary for allowing train to move in safe manner. OBC must be responsible for performing or initiating all closed loop safety validation procedures necessary for assuring safety levels of locomotive subsystem as a whole. Individual subsystems to be validated include: 
- Location system accuracy, availability, and expected failure modes.

- Braking system availability

- Communication system data validation

- Self-tests for proper operation

- Authority limits conform to expected limits

- Positive communications with wayside elements occur at appropriate times, i.e. making sure a crossing warning system starts, or that all wayside inputs from all necessary sources are received and processed appropriately.

- $\mathrm{OBC}$ is also responsible for non-vital functions related to traffic management, i.e., work order reporting, determining pacing speeds, etc.

- $\mathrm{OBC}$ is assumed to be used to provide train-handling instructions to engineer.

\section{Main Control Center (MCC)}

Central Office hardware and software include the tools to provide central and end-ofauthority, work limits, and speed restriction location information to trains and other ontrack equipment like work crews. Software to interpret and display accurate positioning information from the field. (This is both a safety function as well as one that provides improved dispatching). Functions of the RTCS dispatching system include:

- Real-time control of rail freight movements

- Optimized use of resources (infrastructure and rolling stock)

- Online dispatching and rescheduling (Movement Planner)

- Availability of train information for management purposes (data warehouse)

- Train grouping management

- Operational Requirements

- The following elements are displayed on a single screen: preset route, actual route as traveled, different categories of geo-referenced points (driver-specific, company, route-specific).

- Geometric and temporal route management with real-time indication of deviations and time schedule checks (early and late arrivals) for entire route and intermediate stages.

- Automatic generation of trip documents with certificate of route traveled.

- Report or map display of the entire fleet or sub-fleet.

- Management of targets with radius for generating entrance and exit signals.

\section{OVERVIEW OF THE RTCS MODELING PROBLEM}

Papers [4 and 5] present an excellent survey of existing locomotive planning models and algorithms for this problem. There are two kinds of locomotive planning models: Single and Multiple. Single locomotive planning models assume that there is only one type of locomotive available for assignment. These planning models can be formulated as minimum cost flow problems with side constraints. Some papers on single locomotive planning models were made by [1, 2, 3, and 9]. Single locomotive assignment models may be appropriate for certain European railroad companies but are not suited for Egyptian railroad companies since most railroads have multiple types of locomotives. Such multiple locomotive assignment models have been studied by [6, 7 , and 8$]$. The most comprehensive multiple locomotive assignment models were presented in [2 and 9] which takes into account more business rules than any other previous model. This model 
has recently been further generalized and improved by Innovative Scheduling team members and is reported in [10].

This paper focuses on solving RTCS modeling problem which arises at two levels: planning and dynamic assignment. A locomotive plan specifies which types of locomotives will pull each train. The solution of the RTCS planning problem serves as a blue-print to guide day-to-day real-time scheduling, called dynamic locomotive assignment. A locomotive plan gives the "ideal locomotive assignment" in the absence of any disruptions, and the plan is further refined and adjusted in real-time to obtain the dynamic locomotive assignment. Whenever a train arrives at its destination, its set of locomotives or grouping is either assigned to an outbound train in its entirety, or the grouping goes to the pool of locomotives where new groupings are formed. In the former case, we say that there is a train-to-train connection between the inbound and outbound trains and no grouping-grouping takes place. In an ideal schedule, we try to maximize the train-to-train connections of locomotives and thus minimize groupinggrouping. Another important feature of the RTCS planning problem is that the solution should be consistent throughout the week in terms of the locomotive assignment and train-to-train connections. If a train runs five days a week, the locomotive plan should assign the same number and type of locomotives to that train each of the five days it runs. A planned train-to-train connection between two trains should also occur on days that both trains are scheduled to operate.

Consistency of the locomotive assignment and train-to-train connections is highly desirable from an operational point of view.

This paper presents a formulation for the RTCS locomotive planning problem as a Mixed Integer Programming (MIP) problem, which is essentially an integer multicommodity flow problem with side constraints on an underlying flow network. The modeling network groupings of arcs and nodes, where arcs denote trains, nodes denote events (arrivals and departures of trains at terminals), and different locomotive types define different commodities. An important feature of this formulation is that it produces highly consistent locomotive plans.

This formulation can be solved to optimality or near-optimality using the available OR solvers such as QSB+ (Qualification System for Business Plus) and MSIS (Management Science Information System) [11] which include linear and non linear programming, transportation problem, and decision making to model the railway tracking and control. The RTCS planning model easily enables several kinds of what-if analyses which, when conducted manually, are very difficult to carry out. The model can easily assess the impact of proposed train schedule changes on locomotive requirements. A locomotive planning model thus supplements service design. When the service design team changes the operating plan, the locomotive planning model can assess the impact of this change on locomotive requirements. The model will enable service designers to assess modifications in the train plan on locomotive costs.

The RTCS locomotive planning model builds a foundation for future decision support in the real-time locomotive assignment environment. A computerized model for real-time locomotive assignment will significantly reduce train delays due to lack of power. The planning system will generate an optimal plan that becomes an input to the real-time locomotive assignment. The real-time locomotive assignment will adjust the plan while considering current and forecasted system-wide needs and availabilities while also 
adhering to the plan as much as possible. (Fig.2).

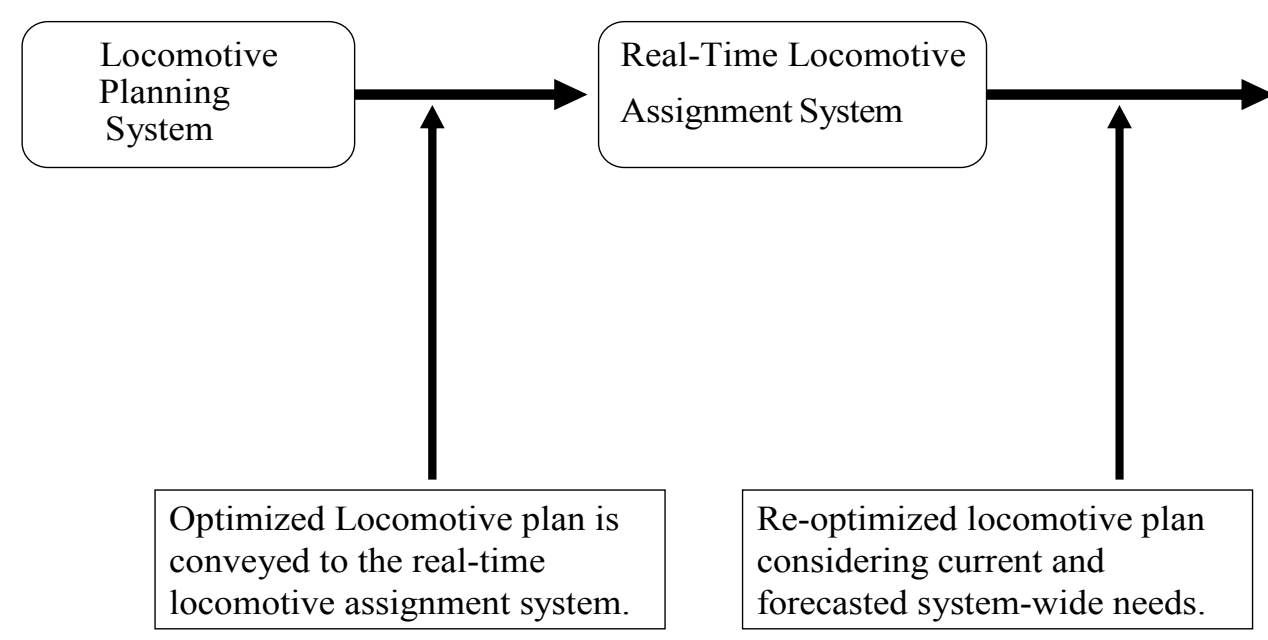

Fig. 2: Roles of RTCS modeling in real time locomotive assignment.

\section{OVERVIEW OF THE RTCS FUNCTIONAL MODEL}

Figure 3 gives an overview of our locomotive planning model. The inputs to the model include geographical data, train schedule and tonnages required, locomotive data, and grouping data. The outputs of the model include train reports, yard reports, and grouping reports. Figure 3 also lists the objective functions and the constraints of the locomotive planning problem.

\section{Formulation of RTCS Modeling Problem}

Consider an RTCS network as shown in fig. 4, where the arcs denote trains, nodes denote events (arrivals and departures of trains at terminals), and different locomotive types define different commodities. An important feature of this formulation is that it produces highly consistent locomotive plans. This formulation can be solved to optimality or near-optimality using the available OR MIP software with large number of decision variables and a comparable number of constraints.

\section{MIP formulation}

Locomotive Data: The railroad authority typically has several different types of locomotives with different pulling and cost characteristics. Denote by $\mathrm{K}$ the set of all the locomotive types and use the index $\mathrm{k}$ to represent a particular locomotive type. Associate the following data with each locomotive type (1) $h^{k}$ : the horsepower provided by a locomotive of type $k € K$ on the arc I $€$ all arcs; (2) $G^{k}$ : the weekly ownership cost for a locomotive of type $\mathrm{k}$; (3) $\mathrm{B}^{\mathrm{k}}$ : fleet size of locomotives of type $\mathrm{k}$, that is, the number of locomotives available for assignment, and (4) $s^{k}$ : an integer variable indicating the number of unused locomotives of type $\mathrm{k} € \mathrm{~K}$. 


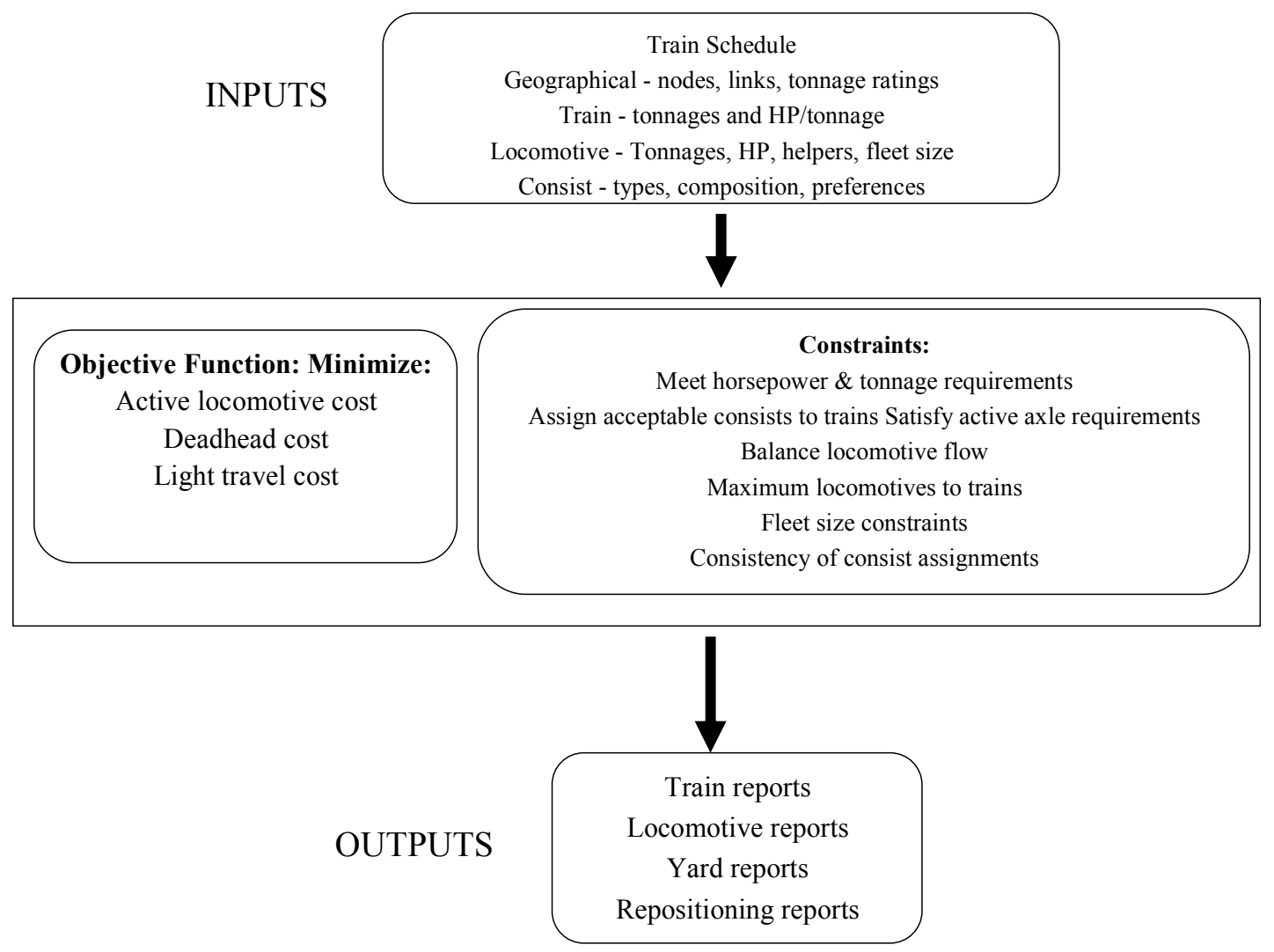

Fig. 3: Overview of locomotive planning model.

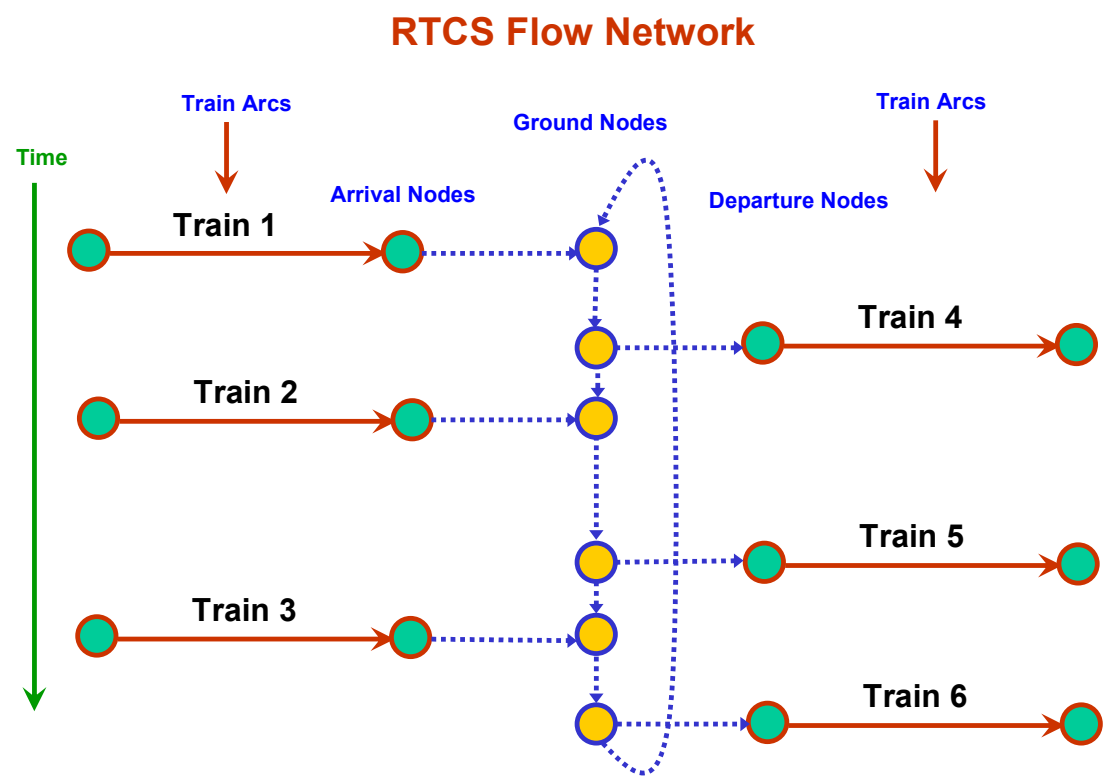

Fig.4: An RTCS Flow network.

\section{Given:}

- A train schedule

- Horsepower and tonnage requirements of trains 
- A fleet of locomotives and their characteristics

- Types of locomotive

Determine:

Assignment of locomotives to trains (groupings)

Satisfying:

A variety of constraints

- Features of the Locomotive Scheduling Problem

- A train is typically assigned multiple locomotives.

- Trains may not run all days of the week.

- Locomotives either actively pull trains or push on them.

- Locomotives can also light travel.

Decision Variables

- Active Locomotives: How many locomotives of each type should actively pull any train $\left(\mathbf{X}_{l}^{\mathbf{k}}\right)$

- Deadhead Locomotives: How many locomotives of each type should deadhead on any train $\left(\mathbf{y}_{l}^{\mathbf{k}}\right)$

Hard Constraints

- Meet the horsepower requirement of every train.

- Meet the tonnage requirement of every train. The solution must be repeatable every week.

Objective Function: Minimize:

- Cost of ownership of locomotives: $\mathrm{C}^{\mathrm{k}}$

- Cost of active locomotive: $\mathrm{d}^{\mathrm{k}}$,

Size of the Network

- More than $\mathrm{N}_{\mathrm{t}}$ trips of trains per day (running different number of days of the week).

- Total of $L_{t}$ Locomotives in the railway network (e.g. Egyptian Railways has about 500 locomotives exported from various countries).

- Each locomotive costs $C_{t}$ in capital cost.

- Each locomotive costs $\mathrm{C}_{0}$ operational cost per year.

\section{RTCS Integer Programming Formulation}

The objective function in locomotive planning is to minimize the total long-term economic cost of operating the entire system. The objective function of the planning model includes the following terms:

- Cost of locomotive groupings actively pulling trains;

- Cost of locomotive groupings deadheading on trains;

- Cost of light traveling groupings; and

- Cost of locomotive ownership. 


\section{Objective Function}

$$
\mathbf{Z}=\quad \sum_{l \in \text { TrainArcs }} \sum_{\mathbf{k} \in \mathbf{K}} \mathbf{C}_{l}^{\mathbf{k}} \mathbf{x}_{l}^{\mathbf{k}}+\sum_{l \in \text { AllArcs }} \sum_{\mathbf{k} \in \mathbf{K}} \mathbf{d}_{l}^{\mathbf{k}} \mathbf{y}_{l}^{\mathbf{k}}-\sum_{\mathbf{k} \in \mathbf{K}} \mathbf{G}^{\mathbf{k}} \mathbf{s}^{\mathbf{k}}
$$

The first term denotes the cost of actively pulling locomotives on train arcs. The second term captures the cost of deadheading locomotives on train arcs and light-travel arcs, as well as the cost of idling locomotives. Included is the variable cost of consist busting in the definition of the term $\mathrm{d}^{\mathrm{k}}$ for each arc I $€$ all arcs. The third term, $\mathrm{G}^{\mathrm{k}} \mathrm{s}^{\mathrm{k}}$, represents the savings accrued from not using all the locomotives.

\section{Decision variables}

There are five types of decision variables in the locomotive planning problem:

- Active Consist Assignment Decisions: Assign an active grouping to each train. The active grouping must provide sufficient tonnage and horsepower to the train.

- Deadhead Consist Assignment Decisions: Determine deadhead groupings traveling on each train.

- Light Travel Consist Decisions: Identify light travel opportunities from power sources to power sinks to reduce locomotive deadheads.

- Train-Train Connection Decisions: Assign groupings from inbound trains to outbound trains at each terminal.

- Locomotive Positioning Decisions: Determine the number of groupings (or locomotives) of each type needed at terminals at the beginning of each day.

\section{Problem set of constraints}

Two categories of constraints: hard constraints and soft constraints. Hard constraints are mandatory constraints which must be satisfied for a locomotive assignment solution to be feasible, whereas soft constraints are flexible and define characteristics which are preferred but not mandatory. Soft constraints may be violated but each such violation incurs a user-defined penalty cost.

- Tonnage power requirement for trains: Each train requires certain tonnage power depending upon the number of cars in the train and the track geometry (i.e., slope of the track) on the route of the train and the grouping assigned to the train must meet this tonnage requirement. Each locomotive type has a given tonnage rating for a train, that is, the tonnages that the locomotive provides to the train, which again depends upon the track geometry. This constraint states that the total tonnage provided by locomotives in the grouping must be greater than or equal to the tonnage requirement of the train it is assigned to.

- Horsepower requirement for trains: Each locomotive provides a certain tonnage and horsepower. The tonnage provided depends upon the locomotive type and the track geometry whereas horsepower (HP) depends only upon the locomotive type.

- Locomotive class to train type: Each train type (e.g., auto train, or merchandise train, or inter-modal train) is targeted to use specific classes of locomotive types.

- Trains with pre-specified power: Some trains may be pre-assigned grouping types and the model is required to honor it.

- Locomotive balance constraints: At any point in time, the number of incoming locomotives of each type entering a station must be equal to the number of outgoing locomotives of that type at that station plus the number of locomotives residing at the station. 
- Minimum connection time constraints: Consists coming from inbound trains must wait for terminal-specific minimum connection time before being assigned to the outbound trains. Typically, the minimum connection time varies from two to twelve hours.

Horsepower Constraints

$$
\sum_{\mathbf{k} \in \mathbf{K}} \mathbf{h}^{\mathbf{k}} \mathbf{x}_{l}^{\mathbf{k}} \geq \mathbf{H}_{l} \quad \text { for all } l \in \text { TrainArcs }
$$

Flow balance at arrival nodes

$$
\mathbf{x}_{l}^{\mathbf{k}}+\mathbf{y}_{l}^{\mathbf{k}}=\sum_{\mathbf{h} \in \mathbf{O}(\operatorname{head}[l])} \mathbf{y}_{\mathbf{h}}^{\mathbf{k}} \text { for all } l \in \text { TrainArcs and all } \mathbf{k} \in \mathbf{K}
$$

Flow balance at departure nodes

$$
\sum_{\mathbf{h} \in(\text { tail[ }[])} \mathbf{y}_{\mathbf{h}}^{\mathbf{k}}=\mathbf{x}_{l}^{\mathbf{k}}+\mathbf{y}_{l}^{\mathbf{k}} \text { for all } l \in \text { TrainArcs and all } \mathbf{k} \in \mathbf{K}
$$

Flow balance at ground nodes

$$
\sum_{l \in[i]} \mathbf{y}_{l}^{\mathbf{k}}-\sum_{l \in \mathrm{O}[\mathrm{i}]} \mathbf{y}_{l}^{\mathbf{k}}=0 \quad \text { for all } \mathbf{k} \in \mathbf{K} \text { and all } \mathbf{i} \in \text { GroundNodes }
$$

Final constraint: All variables are Non-negative and integers.

\section{INPUT AND OUTPUT DATA OF THE RTCS MODELING}

The implementation of the Egyptian RTCS will enable collecting the input data required for the planning of the railway network (routes, trips, stations, trains, and locomotives).

Example of the detailed input data for the locomotive planning includes:

- For the stations: Station ID, station governorate and city, connection time, daily trips

- For the trains: Train ID, type, frequency, horse power requirement, departure station ID, departure route ID, departure day and time, arrival station ID, arrival route ID, arrival day and time.

- For the locomotive: Locomotive ID, fleet size, horse power, total cost, operational cost, and maintenance cost,

Example of the detailed output data expected from the locomotive planning includes:

- For every station: Station ID, arrival train ID, arrival day and time, departure day and time, and trips ID

- For every train: Train ID, locomotive ID and locomotive-group needed for every trip and every day and time

- For every locomotive: Locomotive ID, train ID, station ID, arrival day and time, departure day and time, and trips ID 


\section{CONCLUSIONS}

We first presented in this paper the steps for the implementation of the Egyptian RTCS which shall satisfy the requirements to build an automated locomotive location and management control system and offer effective opportunity to Egyptian Railways authority.

Real time localization of locomotives and trains allow to precisely knowing anytime where locomotives are quickly and effectively communicate with staff, making it possible to get quick and precise decisions. The wide range of mobile communications (GSM, GPRS, EDGE and G3) supported by the three mobile operators allows to choose the best and more efficient communication channel for every application. The accurate sampling of railway roads, locomotives, and trains allows the railway decision makers to evaluate all the costs and to control problems concerning some locomotives (anomalies). Implementation of such a system would achieve the following:

- Improve service reliability for shippers, producing a large benefit for them

- Increase the capacity of routs miles that are now at or above capacity, enabling railroads to avoid a very substantial near-term investment in track and signals

- Produce immediate savings in car and locomotive ownership cost through improved utilization

We presented also in this paper RTCS locomotive planning that determines the set of active locomotives for the scheduled train network, light traveling locomotives from power sources to power sinks, and train-to-train connections. The modeling is based upon mixed integer programming (MIP) formulation which relies on formulating the problem as a multi-commodity flow problem on a space-time network in which each locomotive type represents a commodity and assignment of locomotive to trains is transformed into the flow of trips on a seven-day or weekly space-time train network.

\section{REFERENCES}

1. Ya Goe, Jie Lu Guanagpuan, A bi level model for raiway train set organization optimization, Faculty of IT, university of technology Sydney, Autralia, 2007

2. Artyom Nahapetyan, R. Abuja, F.Zeyney Sargue: A simulation optimization framework for locomotive planning, Gainesville Technology Enterprise Center GTEC, ATMOS,2007 PP. 259-276

3. H.Q. Wang Y.P. Le, L.X. Miao and C. Wang. A bi-level programming model and a solution for public logistics terminals planning. In TRB, 2006, 2006.

4. Warren B. Powell, B.B. Ayari, Approximate dynamic programming for locomotive optimization, Dept of OR and financial Engineering, Princeton University, Princeton ,NJ, October 2006

5. H.Ko, T.Kosek, M.Miyake: Application of dynamic programming to optimization of running profile of a train, Sophia university, Japan, The University of Tokyo, 2005

6. M.H. Wang and Q. Zhao. Optimal model and algorithm of stage plan of intelligent dispatching system for marshalling stations. Journal of the China Railway Society, 27(6):1-9, 2005.

7. C.G. Wang. Research on the model and algorithm of dynamic wagon-flow allocating in a marshalling station. Journal of the China Railway Society, 26(1):1-6, 2004. 
8. S. Dunstall, A. Ernst, H. Jiang, P.Killy, M. Krshnamoorthy: Locomotive restoring with multiple classes and a central dept, CMIS OR-Applications- Locomotive scheduling, CSIRO, September 2004

9. H. Li H.H. Mo F. Shi, Q. Fang and Yong Huang. A bi-level p programming optimization method for the layout of technical service station. Journal of the China Railway Society, (2):1-4, 2003.

10.W.Q. Li and W. Du. Modeling Models and Algorithms for the Daily Working Plan of Railway Technical Station. Southwest Jiaotong University Publisher, Chengdu, 2002.

11. Mohsen Attran, Management Science information System, Johm Wiley and Sons, 1992 